\title{
Official Title
}

National Cancer Institute

\section{Source}

National Cancer Institute. Official Title. NCI Thesaurus. Code C93612.

The formal title of the document. 